\title{
Article
}

\section{SEROPREVALENCE AND CLINICAL PRESENTATION OF CHIKUNGUNYA VIRUS INFECTION AMONG FEBRILE OUTPATIENTS SEEKING HEALTH CARE IN MZUZU CITY, MALAWI}

\author{
Flywell Kawonga ${ }^{1,2,5^{*}}$, Gerald Misinzo ${ }^{1,2}$, Dylo Pemba ${ }^{3}$, Leonard E. G. Mboera ${ }^{2} \&$ Isaac T. Shawa ${ }^{4}$
}

1Department of Veterinary Microbiology, Parasitology and Biotechnology, College of Veterinary Medicine and Biomedical Sciences, Sokoine University of Agriculture, P.O Box 3015, Morogoro Tanzania, gerald.misinzo@sacids.org

2SACIDS African Centre of Excellence for Infectious Diseases of Humans and Animals, Sokoine University of Agriculture, P.O Box 3297 Morogoro, Tanzania; leonard.mboera@sacids.org

3VectorBorne Disease Laboratory, Chancellor College, University of Malawi, P.O Box 280, Zomba, Malawi ,pembadyl@yahoo.ie

4Department of Medical Laboratory Sciences, College of Medicine, University of Malawi, Private Bag 360, Blantyre, Malawi; ishawa@medcol.medcol.mw

5Molecular Biology Laboratory, Mzuzu Central Hospital, P.O Box 209, Mzuzu, Malawi

*Correspondence: flywell.kawonga@sacids.org (F.K)

\begin{abstract}
Chikungunya is a mosquito-borne viral disease caused by Chikungunya virus (CHIKV. We conducted this study determine the seroprevalence and clinical presentation of Chikungunya infection among outpatients seeking healthcare in Mzuzu City, Malawi. Blood samples were collected from malaria negative and non-septic febrile outpatients with fevers $\geq 38^{\circ} \mathrm{C}$, for not more than 5 days. The enzyme- linked immunosorbent assay (ELISA) test was used to detect anti-CHIKV IgM antibodies and its results were used to determine seroprevalence of Chikungunya. A total of 119 serum samples were tested, of these, 73 (61.3\%) tested positive for anti-CHIKV IgM antibodies by ELISA. Laboratory requisition forms were used to capture demographic information such as age, sex, clinical signs and symptoms presented by the enrolled patients. Age groups of 1-9, 10- 19, 20$29,30-39,40-49$, and $\geq 50$ years had $17.8 \%(n=13), 12.3 \%,(n=9), 15.1 \%)(n=11), 19.2 \% ;(n=14), 17.8 \%$ $(n=13)$ and $17.8 \%(n=13)$ proportion of seroprevalence respectively. Most of the CHIKV infected individuals presented with fever $(52.05 \%)$, joint pain (45.21\%) and abdominal pain $(42.67 \%)$. The presence of anti- CHIKV IgM antibodies suggest the presence of recent CHIKV infection and therefore accurate laboratory assays are highly recommended for CHIKV diagnosis and appropriate management of febrile patients.
\end{abstract}

Keywords: Chikungunya, seroprevalence, clinical presentation, febrile outpatient, Malawi

\section{Introduction}

Chikungunya is a viral disease caused by Chikungunya virus (CHIKV) and is transmitted to humans by infected mosquitoes. The name Chikungunya comes from the Makonde language in Tanzania, meaning 'to walk bent over' indicating the painful arthralgia experienced by people infected with CHIKV [1]. Chikungunya, first reported in Tanzania 
in 1952, has since spread across the entire world causing large numbers of epidemics that have infected millions of people in Asia, Europe, the Americas and Pacific Islands. About 40 countries are affected worldwide, experiencing sporadic and epidemic cases [2]. Between 1960s and 1990s, major outbreaks with inter-epidemic periods of 7-20 years have been reported [3].

In Africa, CHIKV infection has been reported in Angola, Burundi, Cameroon, Central African Republic, Gabon, Guinea, Malawi, Mozambique, Nigeria, Republic of Congo, South Africa, Tanzania and Uganda [4, 2, 5, 6]. The largest outbreaks of CHIKV in Africa have been recorded during 2005-2007 in the south-eastern islands of Indian Ocean including the Reunion, Mayotte, Mauritius, the Seychelles and Madagascar [7].

Although CHIKV occurrence in Malawi has been documented [4], its prevalence is not known with certainty. CHIKV infection presents similar signs and symptoms to other diseases such as dengue, malaria, leptospirosis and brucellosis [8]. The highly specific laboratory assays are of paramount importance in differentiating viral infections which greatly present with fever [9]. Hospitals and health care providers in Malawi do not include arbovirus infections as a differential diagnosis among febrile patients. Malaria is often over diagnosed in febrile patients [8]. There is underestimation of the burden for mosquito-borne viral diseases such as Chikungunya, thereby leading to wastage of already scarce resources in Malawi. There is scarcity of published information regarding current prevalence of CHIKV infection in Malawi. This study was carried out to determine seroprevalence of CHIKV outpatients seeking health care in Mzuzu city, Malawi.

\section{Materials and Methods}

\subsection{Study area}

Mzuzu City is located in northern region of Malawi in the wooded and hilly terrain land. It is situated in Mzimba district and its people practice trade and agricultural activities such as tea, rubber and coffee farming. Mzuzu city harbours Mzuzu Central Hospital which is a referral hospital for the northern region of Malawi. It is the biggest health facility in northern region of Malawi and therefore has highest number of visiting patients.

\subsection{Study population}

This hospital-based cross-sectional study was carried out in 2020. The inclusion criteria were outpatients with fever $\geq 38^{\circ} \mathrm{C}$, for not more than 5 days and with symptoms of chills, headache, severe joint pains, dizziness, nausea/vomiting, arthralgia and rash were recruited into the study. Whole blood samples were collected from Malawian consenting participants in order to detect the absence or presence of anti-CHIKV IgM specific antibodies.

\subsection{Data and sample collection}

Outpatients with fevers $\geq 38^{\circ} \mathrm{C}$, for not more than 5 days and with symptoms such as chills, headache, severe joint pains, dizziness, nausea/vomiting, arthralgia, rash were recruited in the study. No restriction to age and gender were considered. Those seriously ill such as in coma and required hospitalisation were not included in the study. In patients were also not included in the study. Laboratory requisition forms were administered by the clinicians to capture demographic information such as age, sex, clinical signs and symptoms presented by the patients.

The outpatients were examined by a qualified clinician at the health facility and enrolled as participants according to the inclusion criteria. Samples of blood $(5 \mathrm{ml})$ were drawn from all consenting malaria negative patients using venipuncture technique by qualified laboratory technologists/technicians and put in ethylene-diamine-tetra-acetic acid (EDTA) and red top plain tubes. Blood sample were at first screened for malaria using 
malaria rapid test, SD Bioline Malaria Ag- Pf/Pan kit (Standard Diagnostics, Suwon city, Republic of Korea). Full blood Count (FBC) tests were also done to rule out sepsis. Malaria negative patients with a non-raised neutrophil count $\left(1.5-8 \times 10^{9} / \mathrm{L}\right)$ were then screened onsite for the absence or presence of anti-CHIKV IgM antibodies using SD Bioline Chikungunya IgM rapid test (Standard Diagnostic, Kyonggi-do, Korea). The sera in plain red top tubes from only positive Chikungunya IgM rapid test results was separated by centrifugation and aliquoted in cryovials. The sera samples were then sent to the The University of Malawi, Chancellor College, Department of Biological Sciences for permanent storage in $-80 .{ }^{\circ} \mathrm{C}$ ultralow freezers until analyzed by enzyme- linked immunosorbent assay (ELISA). The ELISA test results were used to detect anti-CHIKV IgM antibodies to determine seroprevalence.

\subsection{Serological analysis of anti-CHIKV IgM antibodies}

Stored samples were tested for anti-CHIKV antibodies using Abcam's ELISA Kit (Abcam, Cambridge, UK) by strictly following manufacturer's instructions. The diagnostic specificity and sensitivity of the assay was more than $90 \%$. Absorbance of tested samples was read at $450 \mathrm{~nm}$ using an ELISA microwell plate reader (Bio-Rad, California, USA). The results were regarded as positive if the absorbance value was greater than $10 \%$ over the cut off value. The intensity of the product of IgM anti-CHIKV antibodies and precoated CHIKV antigen in microwell was proportional to the amount of CHIKV-specific IgM antibodies in the patient sample. The results were calculated and interpreted in Abcam's unit (NTU) according to manufacturer's instructions. The cut off value in Abcam's unit was $10 \mathrm{NTU}$, negative result was $<9$ NTU and positive was more than $11>$ NTU as per manufactures instructions. Both positive and negative controls contained in the kit were included each assay run to ensure the reliability of the test procedure.

\subsection{Statistical analysis}

Data collected from the clinical history were entered and analyzed using Microsoft Office-Excel 2007 (Microsoft, California, USA) and Epi Info version 7.0.8.0 (CDC, Atlanta, USA). Sex, current fever (fever of $\geq 38^{\circ} \mathrm{C}$ measured by the clinician at the hospital), previous fever (fever presented in patients before the onset of other symptoms at home), abdominal pains, joint pains, bleeding (from the nose and gums), nausea, backache, body weakness, chest pain /backache, dizziness, fast breathing, cough, heart palpitations, itchy legs, leg swelling, redness of eyes, sweating, throat pain and yellow eyes were used to draw frequency tables in relationship to seropositivity of ELISA test. Chi-square independence test using SPSS software was carried to find out if there are any statistical significance of clinical presentations among anti-CHIKV IgM seropositive patients and also statistical significance of observed seropositive results among different age groups ( $\mathrm{p}$ value $<0.05)$.

\subsection{Ethical considerations}

Ethical approval to conduct the study was obtained from the College of Medicine Research and Ethics Committee (P.02/20/2956). All study participants were consented to participate in the study.A written consent was sought and obtained from all adults $(\geq 18$ years) and assent from parents/guardians of children ( $<18$ years old) before recruited into the study. The records of all participants were documented through identification numbers for anonymity.

\section{Results}

\subsection{Demographic characteristics of the study participants}

A total 119 participants who met the inclusion criteria were recruited into the study. Of these, $79(66.39 \%)$ were females and $40(33.61 \%)$ were males. The participant's mean age was 31 years (2-83 years). 
3.2 Seroprevalence of Chikungunya virus infection among age groups

Of the total 119 sera analysed for anti-CHIKV IgM antibodies using ELISA, 73 (61.3\%) tested positive. The highest proportion $(19.2 \% ; n=14)$ of seropositivity among anti-CHIKV IgM positive individuals were observed among individual aged $30-39$ years. Age groups of 1-9, 40-49 and $\geq 50$ years were the second to be detected to have high proportions of anti-CHIKV IgM antibodies. Age of 20-29 years had 11 (15.1\%) cases that were antiCHIKV IgM antibodies positive. Individuals aged group of 10-19 years had the lowest seroprevalence $(12.3 \%, n=9)$. The results showed no any significance difference among age groups in association with the observed seropositive results ( $\mathrm{p}$-value $<0.05)$ (Table 1$)$.

Table 1. The proportion of anti-CHIKV IgM antibodies among seropositive individuals by age group

\begin{tabular}{|c|c|c|c|c|c|c|c|c|c|}
\hline Age group & Number & Age & sinu & erumben & i-Ag & grou & aine & Pennline & Ghi Nus \\
\hline (years) & participa & syea & $\mathrm{CI}$ & barticipl & troes & $C Q$ & bal & iciphgilts & aluec \\
\hline & $(n=119)$ & & ser & o(sitiald9) & & ser & ositit & A9) & sero \\
\hline & & & & & & $(n=$ & & & \\
\hline 1 to 9 & 23 & 1 to & 13 & 23 & 1 to & 13 & 23 & 0.9 & 1.5913 \\
\hline 10 to 19 & 13 & $10 t$ & & 13 & $10 t$ & & 13 & & 9 \\
\hline 20 to 29 & 20 & $20 t$ & 911 & 20 & $20 t$ & 911 & 20 & & 11 \\
\hline 30 to 39 & 20 & $30 t$ & 914 & 20 & $30 t$ & 914 & 20 & & 14 \\
\hline 40 to 49 & 21 & $40 t$ & 913 & 21 & $40 t$ & 1913 & 21 & & 13 \\
\hline$\geq \mathbf{5 0}$ & 22 & $\geq \mathbf{5 0}$ & 13 & 22 & $\geq \mathbf{5 0}$ & 13 & 22 & & 13 \\
\hline
\end{tabular}

(b)

3.3 Seroprevalence and clinical presentation of study participants

In this present study, of the total 119 sera analysed for anti-CHIKV IgM antibodies using ELISA, $73(61.3 \%)$ tested positive. A total of $33(45.21 \%)$ patients who presented with joint pains were positive for anti-CHIKV IgM antibodies. A proportion of $30.14 \%$ with current fever was not seropositive for CHIKV. Patients without abdominal pains $(58.33 \%, n=42)$ had higher seropositivity of anti-CHIKV IgM antibodies than those with abdominal pains $(41.67 \%, \mathrm{n}=30)$. Thirty-eight $(52.05 \%)$ patients with history of previous fever tested positive for anti-CHIKV IgM antibodies. Sixty-four $(87.67 \%)$ patients with no bleeding history were positive for anti-CHIKV IgM antibodies. Majority $(83.56 \%$; $\mathrm{n}=61$ ) of patients without history of vomiting tested positive for anti-CHIKV IgM antibodies. All these results showed no any statistical significance of clinical presentation and anti-CHIKV IgM seropositivity among patients (p-value $<0.05)$ (Table 2$)$. 
Table 2: Clinical presentation of patients with positive anti-CHIKV IgM antibodies by ELISA

\begin{tabular}{|c|c|c|c|c|c|}
\hline Clini- & Partici- & Number & Number of & Chi square & P-value \\
\hline cal & pant's re- & of partici- & anti- & $X^{2}$ & \\
\hline presen- & sponse & pants & CHIKV & & \\
\hline \multirow[t]{3}{*}{ tation } & & $n=119$ & IgM posi- & & \\
\hline & & & tive & & \\
\hline & & & $n=73$ & & \\
\hline Acute & Yes & 34 & 22 & 0.227 & 0.634 \\
\hline \multicolumn{6}{|l|}{ fever } \\
\hline & No & 85 & 51 & & \\
\hline Previ- & Yes & 63 & 38 & 1.892 & 0.169 \\
\hline \multicolumn{6}{|l|}{ ous fe- } \\
\hline \multicolumn{6}{|l|}{ ver } \\
\hline & No & 56 & 35 & & \\
\hline Bleed- & Yes & 12 & 9 & 1.049 & 0.306 \\
\hline \multicolumn{6}{|l|}{ ing } \\
\hline & No & 107 & 64 & & \\
\hline Vomit- & Yes & 20 & 12 & 0.018 & 0.892 \\
\hline \multicolumn{6}{|l|}{ ing } \\
\hline & No & 99 & 61 & & \\
\hline
\end{tabular}




\begin{tabular}{lccccc}
\hline Joint & Yes & 55 & 33 & 0.078 & 0.78 \\
pain & & & & & \\
\hline & No & 64 & 40 & 0.202 & 0.653 \\
\hline Ab- & Yes & 47 & 30 & & \\
dominal & & & & & \\
pain & & & & & \\
\hline & & & & & \\
& No & 72 & 43 & \\
\hline
\end{tabular}

Backache, body weakness, chest pain, fast breathing, itchy legs, leg swelling, nausea, redness at eyes, sweating, throat pain and yellow eyes had a frequency of $1(5 \%)$ each as clinical presentation among anti-CHIKV IgM seropositivity. Chest pain and heart palpitation had a frequency of $2(10 \%)$ each, and dizziness had a frequency of $3(15 \%)$ among antiCHIKV IgM seropositivity.

\section{Discussion}

The study has established the presence of antibodies against CHIKV amongst febrile patients seeking health care at Mzuzu Central Hospital. The presence of anti-CHIKV IgM antibodies suggest the presence of recent CHIKV infection in patients.

The results indicate a high seroprevalence of Chikungunya (61.3\%) as detected by presence of anti-CHIKV IgM antibodies patient's sera. The seroprevalence of Chikungunya in this study is slightly lower but similar to a study in Eastern Sudan, where a seroprevalence of Chikungunya of $73.1 \%$ was reported [10]. The seroprevalence reported in this study is slightly higher but similar with seroprevalence reported among febrile patients in Cameroon and Zambia with a seroprevalence of 51.4\% (anti-CHIKV IgM antibodies) and $36.9 \%$ (anti-CHIKV IgG antibodies), respectively [11, 12]. The difference in seropositivity in this study and other studies may be due to differences in sample sizes and methodologies used. The high seroprevalence in this study is likely to be attributed to the strict inclusion criteria of suspected patients of CHIKV infection whereby patients with malaria and sepsis were excluded for enrolment into the study therefore narrowing down the diagnosis to CHIKV infection and leading to high seroprevalence determined by ELISA assay. This argument is supported by a study done by Kinimi et al., (2018) in Tanzania where they found a higher seropositivity of anti-CHIKV antibodies of $91.75 \%$ among malaria negative patients. However, in the same study in Tanzania a co-infection of $7.4 \%$ of chikungunya and malaria was found [13].

Some of the positivity of ELISA results needs to be carefully interpreted as we may not rule out possibility cross-reactivity of other antibodies. There is a possibility of other arboviruses cross reactivity when IgM antibodies are used for detection of chikungunya [14]. Furthermore, co-infection of DENV, CHIKV and Zika virus among study patients cannot be ruled out from positive results reported in this study. The occurrence of crossreactivity and co-infection of DENV and CHIKV have been reported in Columbia [15]. 
This study has established that diagnosis based on clinical presentation of patients with probable suspicion of CHIKV infection is unreliable. Slightly below half (45.21\%) of the patients who presented with joint pains were positive for anti-CHIKV IgM antibodies and more than half of those without joint pains (54.7\%) were positive for anti-CHIKV IgM antibodies. Although joint pain is the critical symptom for chikungunya infection clinically as its name suggest [16], this study has established that even without joint pain, CHIKV infection is likely to be prevalent in infected patients. This has further shown that some patients do not present joint pains but have CHIKV infections and therefore it is only laboratory analyses that can elucidate the diagnosis of CHIKV infection. This therefore calls for the highly specific laboratory techniques such as serology and molecular nucleic acid amplification for their reliability to differentiate viral and other infections which greatly present with fever [9]. This study has therefore proved the hypothesis that CHIKV is prevalent in Mzuzu city and that people have been exposed to the virus. The study has provided an insight of the clinical presentation of chikungunya among non-malaria and non-septic cases in Mzuzu city, Malawi.

The present study indicates the cases of CHIKV were recent ones through detection of IgM anti-CHIKV antibodies. By detecting anti-CHIKV IgM antibodies, the results suggest a possibility of acute infection. IgM antibodies appear first and can be detected during the first week of the disease particularly from day 5 [17]. This study therefore indicates that CHIKV is prevalent and contributes to the burden of febrile illnesses in Mzuzu city, Malawi. Accurate laboratory assays with high specificity and sensitivity as serological assays are of paramount importance for differential diagnosis of febrile illnesses. The study has also established that there an underestimation of arbovirus infections and misdiagnoses which lead to improper treatments and wastage of already scarce resources for a low income country as Malawi. Therefore, clinicians at Mzuzu Central Hospital should consider CHIKV infection as a differential diagnosis in febrile patients.

\section{Conclusions}

The findings of this study have determined the current seroprevalence status of CHIKV infection in Mzuzu city, Malawi to be high through detection of anti-CHIKV IgM antibodies. Overall, this study therefore concludes that chikungunya is prevalent in Mzuzu city, Malawi and that serological assays are of paramount use in detection of chikungunya infections.

Supplementary Materials: The data used in this study are available from the authors on a reasonable request.

Author Contributions: Conceptualization, F.K., G.M., L.E.G.M.; methodology, FK., L.E.G.M., G.M, D. P., and I.T.S.; validation, L.E.G.M, F.K., D.P., I.T.S and G.M.; formal analysis, F.K and L.E.G.M; data curation, F.K. and L.E.G.M.; writing-original draft preparation, F.K.; writing-review and editing, F.K., L E.G.M., I.T.S and G.M.; supervision, L.E.G.M., I.T.S and G.M. All authors have read and agreed to the published version of the manuscript.

Funding: The study was funded by the Government of United Republic of Tanzania and World Bank (WB ACE II Grant P151847 and Grant PAD1436) through SACIDS Africa Centre of Excellence for Infectious Diseases, SACIDS Foundation for One Health, Sokoine University of Agriculture, Morogoro, Tanzania.

Institutional Review Board Statement: The study was conducted according to the guidelines of the Declaration of Helsinki, and approved by the Ethics Committee of College of Medicine Research and Ethics Committee on $16^{\text {th }}$ march, 2020 (P.02/20/2956).

Informed Consent Statement: Informed consent was obtained from all subjects involved in the study. All study participants were consented to participate in the study.A written consent was sought and obtained from all adults ( $\geq 18$ years) and assent from parents/guardians of children $(<18$ years old) before recruited into the study. The records of all participants were documented through identification numbers for anonymity. 
Conflicts of Interest: The authors declare no conflict of interest. The funders had no role in the design of the study; in the collection, analyses, or interpretation of data; in the writing of the manuscript, or in the decision to publish the results.

\section{References}

1. Robinson M. C. An epidemic of virus disease in southern province, Tanganyika territory, in 1952-1953. Clinical features. Transactions of the Royal Society of Tropical Medicine and Hygiene. 1955, 49, 28 - 32.

2. Wahid, B.; Alia, A.; Rafiquea, S.; Idreesa, M. Global expansion of chikungunya virus mapping the 64-year history. International Journal of Infection Disease. 2017, 58, 69 - 76.

3. Renault, P.; Balleydier, E.; D’Ortenzio, E.; Bâville, M.; Filleul, L. Epidemiology of Chikungunya infection on Reunion Island, Mayotte, and neighbouring countries. Médecine et Maladies Infectieuses. 2012, 42, 93 - 101.

4. Diop, D.; Meseznikov, G.; Sanicas, M. Chikungunya Outbreaks from 2000 to 2015: A Review. MedCrave Online Journal of Public Health. 2015, 2, $1-7$.

5. Antonio, V. S.; Muianga, A. F.; Wieseler, J.; Pereira, S. A.; Monteiro, V. O.; Mula, F.; Chelene, I.; Chongo, I. S.; Oludele, J. O . Seroepidemiology of chikungunya virus among febrile patients in eight health facilities in central and northern Mozambique, 2015-2016. Vector-Borne and Zoonotic Diseases. 2018, 18, 311 - 316.

6. Vairo, F.; Coussoud-Mavoungou, M.P.A.; Ntoumi, F.; Castilletti, C.; Kitembo, L.; Haider, N. Chikungunya Outbreak in the Republic of the Congo, 2019- Epidemiological, Virological and Entomological findings of a South-North Multidisciplinary Taskforce Investigation. Viruses. 2020, 12, 1020; doi: 10.3390/v12091020ss.

7. Pongsiri, P.; Praianantathavorn, K.; Theamboonlers, A.; Payungporn, S.; Poovorawan, Y. Multiplex real-time RT-PCR for detecting chikungunya virus and dengue virus. Asian Pacific Journal of Tropical Medicine. 2012 5, 342 - 346.

8. Waggoner, J.; Brichard, J.; LaBeaud, D. A.; Mutuku, F.; Ndega, B.; Heath, C. J.; Sahoo, M. K. Malaria and Chikungunya Detected Using Molecular Diagnostics Among Febrile Kenyan Children. Oxford University Press, Oxford. 2017, 8pp

9. Cabralcastral, M. J.; Cavalcanti, M. G.; Peralta, R. H. S.; Peralta, J. M. Molecular and serological techniques to detect cocirculation of DENV, ZIKV and CHIKV in suspected dengue-like syndrome patients. Journal of Clinical Virology. 2016, 82, $108-111$.

10. Nahla, M.; Mamoun, M.; Rania, E.; Hadi, M.; Fadilah, S.; Aleanizyg, M. Prevalence and identification of arthropod-transmitted viruses in Kassala state, Eastern Sudan. Libyan Journal of Medicine. 2018 14, 1 - 7.

11. Pistone, T.; Ezzedine, K.; Boisvert, M.; Receveur, M. C.; Schuffenecker, I. Cluster of chikungunya virus infection in travelers returning from Senegal, 2006. Journal of Travel Medicine. 2009, 16, 286 - 288.

12. Chisenga, C. C.; Bosomprah, S.; Musukuma, K.; Mubanga, C.; Chilyabanyama, RM. Sero-prevalence of arthropod-borne viral infections among Lukanga swamp residents in Zambia, PLoS One. 2020, 15, e235322.

13. Kinimi, E.; Bismwa, E. P.; Misinzo, G. Serological evidence of chikungunya and malaria co-infection among febrile patients seeking health care in Karagwe district, Tanzania.Tanzania Journal of Health Research. 2018, 20, 1-8.

14. Lanciotti, R.S.; Kosoy, O.L.; Laven, J.J.; Velez, J.O.; Lambert, A.J.; Johnson A.J. Genetic and serologic properties of Zika virus associated with an epidemic, Yap State, Micronesia. Emerging Infectious Diseases. 2008, 14, 1232 - 1239.

15. Martínez-Pulgarín, D.F.; Chowdhury, F.R.; Villamil-Gomez, W.E.; Rodriguez-Morales, A.J.; Bohm, G.M.;Paniz-Mondolfi, A.E. Ophthalmologic aspects of chikungunya infection. Travel Med. Infect. Dis. 2016, 14, 451-457.

16. Schilte, C. Chikungunya Virus associated long-term arthralgia: A 36 months prospective longitudinal study. PLoS Neglected Tropical Diseases. 2013, 7, e2137.

17. Panning, M.; Grywna, K.; Van Esbroeck, M. Chikungunya fever in travellers returning to Europe from the Indian Ocean region, 2006. Emerging Infectious Diseases. 2008, 14, 416 - 422. 